Cultures \& Conflits

08 | hiver 1992

Les conflits après la bipolarité

\title{
L'après-Mengistu dans la Corne de l'Afrique : une stabilisation impossible?
}

\section{Roland Marchal}

\section{(2) OpenEdition}

1 Journals

\section{Édition électronique}

URL : http://journals.openedition.org/conflits/529

DOI : 10.4000/conflits.529

ISSN : $1777-5345$

Éditeur :

CCLS - Centre d'études sur les conflits lilberté et sécurité, L'Harmattan

Édition imprimée

Date de publication : 6 décembre 1992

ISSN : 1157-996X

\section{Référence électronique}

Roland Marchal, «L'après-Mengistu dans la Corne de l'Afrique : une stabilisation impossible ? »,

Cultures \& Conflits [En ligne], 08 | hiver 1992, mis en ligne le 27 janvier 2003, consulté le 30 mars 2021.

URL : http://journals.openedition.org/conflits/529 ; DOI : https://doi.org/10.4000/conflits.529

Ce document a été généré automatiquement le 30 mars 2021.

Creative Commons License 


\title{
L'après-Mengistu dans la Corne de l'Afrique : une stabilisation impossible?
}

\author{
Roland Marchal
}

1 Il fallait quelque naïveté pour espérer une rapide normalisation de la situation dans la Corne de l'Afrique après l'effondrement du système communiste et la disparition - ou tout au moins la réduction - des enjeux stratégiques dans cette région du monde liée à la fin des tensions Est-Ouest. C'était méconnaître le très profond enracinement local de ces conflits - perceptible déjà par le seul fait de leur durée - et surtout leur autonomisation par rapport aux logiques internationales dues à des facteurs complexes comme la recomposition de ces sociétés par la guerre et les importantes quantités d'armes disponibles pour toutes les aventures militaires ${ }^{1}$. Sans doute, aucune de ces raisons n'était rédhibitoire mais il faut souligner que le nouveau climat international portait également en germe des facteurs de déstabilisation dont deux demeurent tout à fait manifestes dans la Corne de l'Afrique : la disparition d'une cohérence nationaleétatique portée notamment par le modèle soviétique (avec le développement de projets qualifiés de "sécessionnistes") et la nécessité pour de nombreux pays de recycler leurs industries ou stocks d'armement en profitant des opportunités locales (Afrique du Sud, Egypte, Liban, pays de l'Est..) ${ }^{2}$. Avec un peu plus de recul historique, il sera sans doute possible, de mieux décrypter la séquence d'événements qui a conduit à l'effondrement du régime éthiopien, alors qu'il semblait pourtant s'être doté des instruments de sa consolidation : appareil sécuritaire très performant, parti unique, constitution... Il est probable que l'analyse retiendra alors la métaphore d'un phénomène de résonance entre logiques internes et internationales, bien plus que celle d'une induction par l'extérieur, quel que soit par ailleurs le rôle joué par la soudaine modération soviétique face à l'allié d'hier, toujours empêtré dans des conflits sans rémission.

2 L'entrée des maquisards du Front populaire démocratique du peuple éthiopien (FDRPE) dans Addis-Abéba à la fin mai 1991, avec l'aval américain, marquait un tournant radical dans les trajectoires de tous les conflits de la Corne de l'Afrique. C'est en Ethiopie bien 
sûr où il fut le plus directement perceptible car le nouveau pouvoir devait rapidement trouver un mode de réorganisation du champ politique qui donne au moins l'apparence d'un certain pluralisme jugé indispensable pour continuer à bénéficier du soutien diplomatique des Etats-Unis. De façon toute aussi évidente, la chute du régime de Mengistu Haïle-Mariam témoignait également de la victoire du Front populaire de libération de l'Erythrée (FPLE) qui apparaissait sans doute plus que le FDRPE comme le véritable maitre d'oeuvre des offensives menées depuis près de trois ans et qui firent basculer le pouvoir à Addis-Abéba, alors que ce dernier avait tenu bon pendant trois décennies contre les indépendantistes érythréens appuyés à partir de la fin des années 1970 par ses épigones éthiopiens. Ce succès était optimal pour les nationalistes érythréens sur deux plans. Le FPLE avait provoqué d'une part une crise de régime à Addis-Abéba, les nouveaux détenteurs du pouvoir dans la capitale éthiopienne demeuraient cependant largement dépendants de lui tant au niveau politique que militaire ${ }^{3}$. D'autre part, en Erythrée même, le FPLE retirait, seul, une légitimité accrue d'une victoire, qu'il avait obtenue sans s'allier avec d'autres organisations érythréennes dont la réalité opérationnelle et les capacités militaires étaient par ailleurs toutes relatives.

3 L'analyse commune a fait des Etats-Unis les parrains de ce nouvel ordre régional, puisqu'après avoir porté sur les fonds baptismaux le nouveau régime à Addis-Abéba et noué d'excellentes relations avec Asmara, ils n'ont pas ménagé leur soutien depuis, tant au niveau interne (lors des élections régionales de juin 1992 en Ethiopie) qu'au sein des instances internationales comme le Fonds monétaire international (lors des négociations sur la dévaluation du birr). Pourtant, cette perception fait peu de place au véritable gagnant au niveau régional: le Soudan. Cet Etat n'a pas ménagé ses efforts pendant des années pour affaiblir l'Ethiopie. Son armée avait déjà mobilisé bien avant la prise de Massawa en février 1990, tournant décisif dans la guerre, ses moyens logistiques et ses services de renseignements pour aider les insurgés à écraser les forces gouvernementales éthiopiennes ${ }^{4}$. Les dividendes politiques et militaires d'une aide aussi massive allaient être multiples. Comme nous allons le voir, la crise de l'Armée populaire de libération du Soudan/Mouvement populaire de libération du Soudan (APLS/MPLS) dirigée par John Garang en constituait un de taille, mais ce n'était pas le seul. La victoire du FDRPE permettait de plus au régime soudanais, pourtant isolé sur la scène internationale et en proie à des difficultés économiques internes considérables, d'accéder à une reconnaissance régionale dont il n'avait pas bénéficié depuis près de 20 ans. Il y a quelque ironie à souligner cette convergence d'intérêts entre les Etats-Unis et le Soudan islamiste, mais on aurait tort de n'y voir que du malice.

De façon plus ambiguë, ces changements radicaux affectèrent également Djibouti où certaines parties de l'opposition afar se retrouvèrent alors dans une posture complètement nouvelle, la lutte armée devenant une option moins irréaliste que dans la période antérieure. En l'espace de quelques mois, plusieurs des difficultés qui avaient miné les tentatives de luttes armées vers la fin des années 1970 (lors de la constitution du Front démocratique de libération de Djibouti) étaient levées: l'armement était disponible en quantité et en qualité; l'effondrement de l'appareil d'Etat éthiopien permettait la constitution d'un sanctuaire accessible sans condition politique forte ; la dissolution de la milice afar de la région autonome d'Assab offrait de réelles possibilités de recrutement de cadres expérimentés. Il serait erroné cependant de faire de l'opposition armée djiboutienne un appendice des Afar d'Ethiopie. Si leur participation au Front pour la restauration de l'unité et de la démocratie (FRUD) est aujourd'hui 
assurée, ce mouvement a ses racines dans le blocage de la vie politique à Djibouti et l'internationalisation relative du FRUD doit être analysée également comme le pendant d'une évolution analogue de l'Armée nationale djiboutienne.

5 Le cas somalien n'est pas le moins inintéressant avec les implications des transformations en Ethiopie sur la situation somalienne tant au Sud du pays où les forces de Congrès de la Somalie unifiée (USC) se battaient alors contre les restes de l'armée du dictateur déchu et des milices des clans identifiées à son destin, qu'au Nord où le Mouvement national somalien (MNS) décidait la séparation de l'ancienne colonie anglaise, le Somaliland, de l'Etat somalien et la constitution d'une nouvelle entité étatique dont la légitimité internationale s'appuyait sur l'histoire coloniale, comme pour l'Erythrée. Le Somaliland déclara son indépendance le 26 juin 1960 et attendit jusqu'au premier juillet pour fusionner ses institutions avec celles de l'ancienne colonie italienne devenue indépendante ce même jour.

6 Plutôt que de survoler ces différents conflits ${ }^{5}$, il est plus intéressant de se consacrer à une analyse un peu fouillée de deux cas significatifs dans la conjoncture actuelle: le conflit au Sud-Soudan avec l'émergence d'une faction sécessionniste au sein de l'A.P.L.S. et la réorganisation du champ politique en Ethiopie liée au règlement de la question oromo.

La fragmentation du mouvement insurgé sud-soudanais.

7 L'effondrement du régime de Mengistu Haïle-Mariam à la fin mai 1991 a eu des conséquences désastreuses pour l'APLS 6 , à commencer par la perte de son sanctuaire et, donc, le quasi-effondrement de son système logistique. Ces effets se faisaient rapidement sentir et provoquaient l'affaiblissement de la coordination entre les différents contingents de l'APLS au Sud-Soudan. La perte de la radio qui émettait à partir d'Addis-Abéba vers le Soudan allait se révéler l'un des aspects les plus négatifs de ce retournement de situation. Elle constituait de loin le principal atout politique de l'APLS $^{7}$ dans ses rapports avec l'opposition nord-soudanaise alors complètement marginalisée par la répression extrêmement efficace et brutale des islamistes à Khartoum. En effet, la radio offrait tout à la fois une tribune pour la critique du régime à un moment où une bonne partie de la population doutait encore de la nature réelle du pouvoir mis en place après le coup d'Etat de juin $1989^{\circ}$, une possibilité de contourner le contrôle extrêmement strict des moyens d'information au Nord-Soudan, le moyen d'agréger la lutte menée par l'APLS à celle de l'opposition nordiste et de crédibiliser ainsi aux yeux d'une opinion publique particulièrement rétive la convergence politique entre mouvements politiques traditionnels et opposition armée sud-soudanaise.

8 Ce départ précipité d'Ethiopie, qui ressemblait plus à une fuite éperdue voire à une déroute, posait déjà un premier problème politique à la direction du mouvement. Certains ne comprenaient pas pourquoi John Garang s'était obstiné dans son aveuglement: non seulement, il n'avait pas organisé le retrait en bon ordre de ses troupes du territoire éthiopien mais il avait refusé jusqu'au dernier moment d'avoir des contacts avec le FDRPE et le FPLE; or ces deux organisations n'étaient pas fondamentalement hostiles au niveau des principes à certaines revendications des sudsoudanais, quand bien même leur dépendance vis-à-vis de Khartoum rendaient les résultats d'une telle démarche particulièrement aléatoires ${ }^{9}$.

9 Les conséquences furent tout aussi négatives pour les 400.000 réfugiés sud-soudanais chassés de leurs camps dans la zone de Gambella et d'Itang par les combats provoqués par le Front de libération oromo (FLO) qui entendait, sans doute sur la suggestion de 
Khartoum qui ne lui avait pas ménagé son soutien dans les années précédentes, prendre sa revanche sur l'APLS.. En effet, depuis sa création en 1983, l'APLS opérait dans la région éthiopienne de Gambella et avait installé une base à Assossa à partir de laquelle les insurgés sud-soudanais menaient des actions militaires dans la région du Nil Bleu où de nombreux réfugiés éthiopiens, notamment oromo, s'étaient installés. Un camp du FLO à Yabus fut attaqué une première fois en 1988, mais ce fut surtout en septembre-novembre 1989 que l'APLS réussit à détruire de nombreuses infrastructures de l'organisation oromo ${ }^{10}$. On comprend donc l'animosité de ces derniers. Il parait de plus assez évident que des deux côtés de la frontière, les gouvernements ont réussi avec succès à instrumentaliser les mouvements armés ${ }^{11}$ et que ces derniers ont souvent multiplié les exactions contre les populations civiles de l'autre côté de la frontière, parce qu'ils se sont retrouvés pris dans des conflits de localité peu liés à leur lutte spécifique.

Ce reflux de réfugiés vers la zone de Nassir au Soudan constitua l'un des facteurs qui contribuèrent à la division du mouvement. En effet deux problèmes se posèrent rapidement dès leur retour au pays. En exil, les "instruits" avaient mis en place des structures d'administration civile dans les camps de réfugiés qui leur octroyaient de réels pouvoirs. De retour au Soudan, ils se retrouvaient face à des combattants de l'APLS qui n'avaient aucune envie de respecter des prérogatives qui n'existaient nulle part ailleurs dans les zones dites libérées et qui ont donc fait jouer la force en leur faveur. Cette contradiction déjà importante, qui soulève un problème essentiel sur lequel nous allons revenir, fut encore aiguisée par une série de coïncidences. En effet parmi ces réfugiés se trouvaient des Dinka, originaires de la province d'Aweil, qui considéraient avec hostilité le mouvement de John Garang, jugeant notamment que ce dernier n'avait rien fait lorsque les milices pro-gouvernementales avaient dévasté leur région. De plus, le dénuement de ces migrants était extrême et l'aide humanitaire qu'ils espéraient de la part de l'APLS n'arriva pas. La mort en août 1991 du général Pio Yukwan, membre du Conseil de commandement révolutionnaire (organe dirigeant de la junte militaire à Khartoum) et originaire de la zone de Nassir suscita également la croyance parmi les cadres de l'APLS que l'accalmie dans les combats pourrait être bien plus courte que prévue dans cette région et que le coût humain allait être très élevé.

La question de la sécession vint se surajouter à l'ensemble de ces problèmes. La revendication sécessionniste n'apparaissait plus comme un tabou politique, parce qu'elle pouvait s'appuyer sur des précédents légitimes ou quasi-légitimes, aux yeux des occidentaux, en Europe de l'Est et dans la Corne de l'Afrique (Erythrée, Somaliland). La séparation du Sud-Soudan à laquelle aspirent les dissidents de l'APLS est un thème récurrent du discours politique depuis l'indépendance. D'abord, la base de la guérilla a toujours été largement acquise à l'idée d'une séparation. Les raisons d'une telle attitude ont certes varié ; mais affirmer, comme le firent les dissidents, une préférence pour la sécession ne pouvait que leur faire gagner la sympathie d'une population qui éprouve quelque difficulté à comprendre le contenu du "nouveau Soudan" proposé par John Garang, alors même que l'armée et les milices gouvernementales poursuivent prédations et massacres. Les sécessionnistes optèrent pour cette attitude d'autant plus facilement que la position américaine depuis le renversement de Mengistu n'était pas dénuée d'ambiguïté. De nombreux observateurs soudanais sont convaincus, non sans bonnes raisons, que les Etats-Unis tiennent jusqu'à présent un double discours, affirmant l'unité nécessaire du pays mais adoptant dans leurs propositions de 
règlement un échéancier qui autorise pour une période significative une quasiséparation du Sud d'avec le Nord du pays .

Une autre facette mérite également attention: le doute de plus en plus grand qu'éprouve les Sud-Soudanais sur la sincérité des hommes politiques de Khartoum, quelle que soit leur appartenance partisane. La manière dont les accords d'Addis-Abéba ont été rompus par le président Nimeyri, les manoeuvres dilatoires des grands partis traditionnels après 1986 pour ne pas abroger "les lois de septembre" (i.e. la législation islamique imposée par la dictature militaire en septembre 1983) ont alimenté cette méfiance croissante. Les Sud-Soudanais ont également le sentiment de faire seuls les frais de la guerre actuelle. C'est leur région qui est dévastée par la guerre, leur population massacrée, leurs enfants privés de formation, accroissant d'autant le différentiel de développement entre les deux parties du Soudan.

13 La division de l'APLS en deux tendances, dites de Nassir et de Torit, à la fin août 1991 se fit également dans un cadre politique général tout à fait délétère à l'intérieur du mouvement et qui peut être analysé à partir de plusieurs faiblesses structurelles récurrentes depuis le début de la guerre en 1983: un fonctionnement interne plus militaire que politique, une sous-politisation de la guérilla, une importance accrue des problèmes ethniques liés à l'attitude de J. Garang et à l'absence de vision politique de ses cadres.

En effet, les origines de ce mouvement de guérilla restent fondamentalement attachées à une mutinerie de certains contingents de l'armée. L'APLS s'est constituée, pour l'essentiel, à partir de deux groupes. Le premier est formé de guérilleros Anya-nya des années 60 , intégrés dans l'armée nationale après les accords de paix d'Addis-Abéba en 1972, qui prirent le maquis à Akobo dès 1975. Après quelques embuscades, ces soldats perdus, qui revendiquaient le nom d'Anya-nya II, se réfugièrent en Ethiopie ; ils y ont vivoté jusqu'en 1983 où ils furent rejoints par d'autres militaires, officiers et soldats, qui abandonnèrent individuellement leur poste. Le second groupe est constitué par la grande majorité des recrues des bataillons 104 et 105 stationnés à Bor, qui se révoltèrent au printemps 1983. Les dirigeants de ces deux factions étaient donc des militaires, bien que des politiciens aient figuré dès cette période dans leur entourage.

Alors que la faction Anya-nya II préférait d'emblée une sécession ou une fédération sans réelle consistance, l'autre tendance conduite par John Garang s'y refusait sachant que l'option séparatiste ne pouvait que rendre plus difficile la quête de soutiens internationaux, notamment de la part de l'Ethiopie de Mengistu Haïle-Mariam. Surtout, John Garang refusa une différenciation très nette des rôles politique et militaire au sein du mouvement. Derrière ces questions importantes d'organisation transparaissaient en fait les termes d'un débat récurrent depuis plusieurs années parmi les opposants sudsoudanais sur l'échec du mouvement Anya-nya I dans les années 60. Anya-nya I ne réussit à adopter un fonctionnement centralisé qu'en 1970 sous la houlette de son chef militaire, Joseph Lagu, qui marginalisa autoritairement les politiciens. Cette défiance vis-à-vis des civils, présumés apporter divisions tribales, polémiques absconses et corruption, se retrouve avec la même intensité dans l'APLS. Certes, le gouvernement de Khartoum sut nourrir ces dissensions grâce à certains hommes politiques sudistes dévoués, qui acceptèrent la remise en cause des accords d'Addis-Abéba puis l'organisation de milices tribales opposées à l'APLS, dans l'espoir de gagner quelque promotion. 

sensibles au vernis marxiste du manifeste de l'APLS/MPLS, les autorités éthiopiennes ont préféré parier sur John Garang, réputé bon officier, doté d'un certain charisme et connu pour sa fermeté. L'attaché militaire éthiopien à Khartoum ne ménagea pas ses efforts pour convaincre les opposants sudistes les plus hésitants que cet ancien capitaine du mouvement Anya-nya I, qui avait obtenu un doctorat dans une Université américaine, était vraiment l'homme de la situation. Leur aide fut également décisive dans plusieurs crises qui affectèrent l'APLS, notamment dans la mise à l'écart en 1988 du numéro deux de l'organisation, Karabino Kuanyin Bol, qui commandait la place de Bor avant de passer à la dissidence en 1983.

Ce caractère martial de l'organisation, censé être le meilleur antidote aux comportements politiciens ou ethniques, a donc été cultivé avec constance et a considérablement contribué à une sous-politisation de la guérilla. S'il lui a permis de subsister, sous une forme relativement unifiée jusqu'en août 1991, il a induit de nombreux effets pervers. D'abord, la militarisation de la vie interne du mouvement a considérablement réduit l'espace laissé au débat politique. Le manifeste publié en 1983 est le seul texte programmatique après plus de huit années de combat. La mise en forme d'intuitions intéressantes sur le "nouveau Soudan"12, à commencer par les futurs rapports entre pouvoir central et entités régionales, est restée à un stade excessivement idéologique. La réflexion sur l'organisation du champ politique, notamment au Sud du pays, fut tout aussi embryonnaire. Ensuite, la guerre contre Khartoum étant l'enjeu unique, la mise en place de nouvelles structures d'administration et d'éducation dans les zones libérées n'était plus qu'un faire-valoir utile pour les contacts avec les organisations humanitaires et laissée pour le reste à la bonne volonté de certains cadres intermédiaires. Aussi, le pire y côtoie le meilleur, compte tenu des faibles moyens dont dispose cette guérilla. Il en découle une conséquence importante : la constitution de groupes combattants et leur entretien ont été très longtemps des priorités absolues. Le recrutement forcé, notamment de jeunes adolescents, n'est certes pas une spécificité sud-soudanaise. Les Erythréens, les Tigréens et, à une échelle bien plus grande, le gouvernement d'Addis-Abéba y ont eu recours sans aucun état d'âme. Mais cet usage répété de la coercition augure mal du Soudan démocratique et laïc, sans cesse promis par la propagande de l'APLS. Tout aussi graves, mais la responsabilité des dirigeants de l'APLS est là plus difficile à mesurer, sont les violations permanentes des droits de l'homme (massacres, pillages, détentions arbitraires, mauvais traitements...). Les cas ont été fréquents dans l'Est-Equatoria mais certains groupes nuer ou dinka n'y ont guère échappé non plus. Ces violations furent souvent le fait de chefs militaires qui, issus d'un terroir, mettaient en oeuvre leurs moyens militaires pour intervenir dans des conflits locaux sans rapport avec la confrontation entre le gouvernement de Khartoum et la guérilla. Il faut souligner les énormes difficultés de communication, qui rendent très lentes les interventions de la direction du mouvement, lorsque celle-ci essaie d'y mettre fin. Un autre aspect tout aussi dangereux de cette option militariste réside dans la stratégie adoptée. L'APLS a miné des centaines de kilomètres de pistes sans tenir compte le moins du monde des populations pour qui ces voies de communications demeuraient vitales. On trouve, dans certains discours de responsables de la guérilla, l'idée que cette guerre, pour amener une véritable solution, devra être meurtrière. 
18 La dimension ethnique est particulièrement importante et dans le même temps très difficile à analyser car il faut tenir compte d'éléments politiques ou sociologiques (comme la différence entre militaires et civils) qui ont joué un rôle important dans les prises de position depuis la création du mouvement. John Garang a insisté dès le début de la crise sur cette dimension qui aurait été instrumentalisée par les dirigeants de l'autre faction pour tenter de le marginaliser. Certes, la personnalité des leaders ${ }^{13}$ et les zones du Haut-Nil dans lesquelles la scission se limita pendant plusieurs mois, donnaient quelque cohérence à cette version : on peut en effet faire référence à des tensions anciennes entre Nuer Jikany et Mor Lou d'un côté, et les autres Nuer de la province de Jonglei de l'autre ou, sur une autre mode à l'alliance Shilluk-Nuer contre Dinka. Mais, il faudrait également souligner l'intensité de ces problèmes avant la crise du mouvement car elle explique pour une part les difficultés que l'APLS a rencontrées lors de son installation dans la province de l'Equatoria: le poids des proches de J. Garang et de façon plus général des Dinka de Bor (d'où est originaire J. Garang) a suscité des mécontentements et des tensions très fortes qui se sont manifestés sous de multiples formes depuis un an. Ainsi des Dinka du Bahr el-Ghazal conduit par Dhol Acuil, le lieutenant Ajing Adiang et le capitaine Benjamen Atem, ont mené une fronde contre Garang l'accusant de les envoyer toujours sur le front afin de préserver les Dinka de Bor. A cette critique s'ajoutait l'amertume liée à l'exécution de Benjamen Bol, ancien représentant de l'APLS à Londres, lors d'un séjour à Addis-Abéba. ${ }^{14}$ Le régime de Khartoum a su profité de cette division dans un mouvement dont l'unité constituait une force et une différence peu communes avec les partis politiques classiques en facilitant pendant de longs mois l'approvisionnement de la faction de Nassir. Sa stratégie était remarquable et a failli réussir. Elle était construite en trois étapes : isoler d'abord la tendance de Torit (i.e. de John Garang) en discutant avec l'autre tendance et en menant campagne au niveau international contre les "comportements dictatoriaux" (sic) du leader sud-soudanais. Ce soutien diplomatique au groupe de Nassir devait être complété par une aide alimentaire et sans doute militaire pour combattre la faction rivale dans tout le Sud-Est du Sud-Soudan. Deuxièmement: mener une offensive militaire contre l'APLS de Torit sans chercher la confrontation avec l'autre tendance et lui briser les reins ; c'est ce qui s'est fait à partir de la prise de Pochalla au début mars 1992. Troisième temps : accepter tambour battant la médiation nigériane et arriver à la conférence d'Abuja face à deux tendances affaiblies par des affrontements fratricides et disposées à entériner un accord largement dicté par Khartoum.

19 Ce plan a bien failli fonctionner mais Khartoum a commis une erreur qui est traditionnelle dans les relations entre politiciens du Nord et du Sud : les islamistes ont largement sous-estimé l'intelligence tactique de leurs adversaires et leur lucidité sur les conséquences d'une trop grande complicité avec eux. Certes, la crédibilité du groupe de Lam Akol fut rapidement limitée par une accumulation de choix politiques privilégiant des alliances avec les islamistes du Nord du pays et surtout par la récurrence de massacres de Dinka dans les localités de Kangor, Bor, Yirol, Rumbek, Tonj et Gogrial entre septembre 1991 et mars 1992, qui relativisaient fortement les critiques formulées sur l'autre faction, d'autant que ces tueries n'étaient pas exclusives d'autres violations des droits de l'homme comme le vol de bétail et des biens, le déplacement forcé de villages entiers. Certains responsables du groupe, plutôt atterrés par ces alliances politiques ou le comportement de leurs troupes sur le terrain ont préféré se distancier du mouvement armé, comme les commandants Achol Marial, Deng Ayen, et Telar Ring ${ }^{15}$. Derrière la polémique entre les deux factions on retrouve à 
vrai dire des problèmes récurrents de la guérilla sud-soudanaise depuis sa naissance, à savoir la multiplication des exactions et opportunisme total dans la politique locale.

20 L'offensive de l'armée gouvernementale contre le mouvement de John Garang fut un succès même si elle commença tardivement et fut particulièrement meurtrière pour toutes les parties en présence. Mais la junte militaire avait parié trop vite sur un essoufflement rapide des combattants de l'APLS et n'avait pas escompté un rapprochement des deux tendances au moment de la conférence d'Abuja en juin 1992, rapprochement qui fit capoter sa stratégie politique ne lui laissant plus que l'option militaire.

21 Depuis cette date, les événements se sont précipités au Sud-Soudan. D'une part, la junte islamiste connaît d'importantes difficultés au niveau international à cause de la répression que mène son armée dans la région de Juba ${ }^{16}$ après l'attaque lancée par l'APLS en juillet 1992. D'autre part, le mouvement de John Garang connaît une nouvelle crise avec la dissidence de William Nyuon Bani, qui était l'adjoint de John Garang depuis la division de l'APLS en août 1991, mais qui avait beaucoup contribué à réunifier les deux factions lors des discussions d'Abuja en circonvenant les partisans d'un ostracisme de la faction de Nassir. Comme les autres dissidents, il bénéficie d'une certaine aura comme militaire dans le mouvement et son départ crédibilise aux yeux des plus hésitants les vives critiques sur le comportement dictatorial de John Garang. Il est difficile de mesurer actuellement les implications de cette nouvelle division sur les combats qui se poursuivent autour de Juba d'autant que, d'un point de vue ethnique, William Nyuon Bani est Dinka par naissance et Nuer par "résidence et affiliation". Il est cependant sûr que la situation de John Garang s'est nettement effritée dans le Bahr elGhazal et l'Equatoria. La nomination de Kuol Manyang Juuk en remplacement de William Nuyon Bani risque de poser rapidement de nouveaux problèmes. Cet homme est connu depuis plusieurs années pour les mauvais traitements infligés aux populations civiles et entretient de très mauvaises relations avec les Latuka d'Equatoria, parmi lesquels est recrutée une partie importante des combattants de l'APLS de cette région, depuis qu'il a giflé lors d'une vive altercation l'évêque de Torit qui est également un notable de cette communauté.

22 Le groupe de Nassir ne peut qu'être satisfait de ces nouveaux développements: sa stratégie actuelle est de contribuer à une fragmentation de l'autre faction en espérant pouvoir à terme renouer des alliances avec les opposants en jouant non sans démagogie sur des accords locaux ou sur la peur omniprésente d'une domination Dinka. Pourtant sa crédibilité n'est pas au plus haut malgré l'attaque surprise de Malakal en novembre 1992 : l'aide patente du gouvernement à certains moments, le comportement tout aussi prédateur de son aile militaire et les problèmes politiques internes diminuent d'autant la portée de ses critiques à l'encontre du mouvement rival et relativisent ses prétentions démocratiques. Sa position radicale sur l'indépendance du Sud-Soudan serait également moins ferme qu'il n'avait semblé à un certain moment et dépendrait largement de la configuration de l'opposition au Nord du pays ou de l'attitude des grandes puissances.

23 L'APLS est donc aujourd'hui à un carrefour de son histoire. Deux scénarios semblent s'imposer, sans qu'il soit aujourd'hui possible de choisir l'un plus que l'autre: la fragmentation de cette organisation en petites bandes de bandits ruraux ou une recomposition relativement rapide d'un mouvement d'où John Garang serait éventuellement exclu. La première trajectoire correspondrait à une situation analogue 
à celle qu'avait connue le Sud-Soudan entre 1963 et 1970. Il avait alors fallu une aide massive d'Israël et le sanctuaire ougandais pour permettre une réorganisation et une centralisation des groupes rebelles. Le second scénario n'est pas improbable mais dépend de variables difficiles à évaluer. L'impact du départ de William Nyuon Bani, le résultat des combats autour de Juba, la mobilisation internationale contre le régime de Khartoum, l'attitude de l'Ethiopie face à la stratégie islamiste de son voisin, l'évolution de l'opposition nordiste tant il est vrai que, si l'opposition au Sud a besoin d'unité, celle au Nord devra se construire une nouvelle crédibilité...

La question oromo et la recomposition politique en Ethiopie

24 L'autodétermination des nationalités en Ethiopie, quelquefois rebaptisée régionalisme ethnique, est aujourd'hui défendue par le FDRPE contre toute la tradition politique éthiopienne (mais aussi une bonne partie de la tradition des études éthiopiennes ${ }^{17}$ ). Certains observateurs font cependant remarquer que ce principe tend à devenir de plus en plus abstrait au fur à mesure que l'assise du nouveau pouvoir s'affermit. Sans dénier cet aspect de la réalité, il faut admettre que cette politique est motivée par plusieurs raisons qui tiennent à la fois à la trajectoire historique de l'opposition armée dans ce pays et à des raisons plus conjoncturelles liées notamment au nouveau contexte international et à la prime à la démocratie offerte par les Etats-Unis dans ce nouvel ( ?) ordre international.

Tout d'abord, l'affirmation des droits des nationalités traduit sans doute la fin d'un cycle historique de la contestation en Ethiopie. En effet, depuis le milieu des années 60 , pratiquement toutes les organisations politiques se sont construites comme des tentatives de solution au problème des nationalités ${ }^{18}$. On aurait donc tort de ne faire de cette question qu'un stratagème idéologique ou un idiome politique particulier. La dynamique actuelle et les nouveaux positionnements des différents groupes éthiopiens - à commencer par le FDRPE lui-même- attestent d'ailleurs la prééminence du sentiment national sur d'autres choix idéologiques ou politiques. Par exemple, la principale composante du FDRPE, le Front populaire de libération du Tigray (FPLT), a été créée en 1974 comme l'expression d'un double constat. D'une part, les organisations politiques "multinationales" ou pan-éthiopiennes comme Meïson ou le Parti révolutionnaire du peuple éthiopien (PRPE) restaient dominées intellectuellement par des "Amhara" et donc par une vision centralisatrice et "chauvine" de l'Ethiopie. D'autre part, il n'existait pas d'expérience politique commune suffisamment significative pour mener la lutte contre le Derg sur une base transcommunautaire. Outre sa méfiance (qui a perduré) vis-à-vis des organisations politiques non strictement nationalitaires, le FPLT a hésité sur le contenu de ses propres revendications. Ainsi, au début 1976, il se prononça pour la sécession du Tigray. Il fallut les pressions conjointes d'une fraction de son appareil militaire et du Front populaire de libération de l'Erythrée (FPLE), peu désireux de voir le cas érythréen perdre sa spécificité, pour que ce programme évoluât vers une autodétermination dont on laissait entendre, sans toutefois le dire, qu'elle pourrait aller jusqu'à la sécession. Pendant plus d'une décennie, le FPLT a donc fonctionné comme une organisation régionaliste sans visée explicite sur le contrôle du pouvoir central. Cette stratégie n'a été remise en cause que très tardivement avec la création du FDRPE en janvier 1990, qui n'a pas toutefois induit de transformations substantielles dans l'énoncé des revendications nationalitaires du FPLT et, donc, du FDRPE. 
Une seconde motivation était plus conjoncturelle. Il fallait marginaliser l'ancienne classe politique accusée globalement de tous les maux sans faire l'analyse des raisons de son implication réelle dans le jeu politique et dans les mécanismes de prise de décision, quitte également à leur préférer des personnages de l'époque impériale qui avaient été fustigés 15 ans auparavant pour leur corruption et leur népotisme. Près de 1500 personnes sont détenus depuis juin 1991, certes dans de bonnes conditions. En imposant un tel choix, on pénalisait fortement la seconde nationalité du pays, à savoir les Amhara, quelque peu diabolisés dans les premiers mois du nouveau régime notamment parce qu'ils étaient très bien représentés dans les instances du Parti des travailleurs éthiopiens (PTE), même si ce n'est pas au niveau décrit par la rhétorique du FPLT. Cette nationalité ne peut guère se reconnaître aujourd'hui dans le Premier ministre amhara, Tamrat Layne, et dans les responsables de son Mouvement démocratique du peuple éthiopien, partenaire principal du FPLT au sein du FDRPE, mais marginal dans la société amhara, tant urbaine que rurale. De plus, c'est cette communauté qui fournissait la majorité des fonctionnaires et, plus justement, la culture et la langue de l'appareil d'Etat: si on ne naissait pas Amhara, on pouvait le devenir pour peu qu'on adoptât dans la sphère publique les normes et les comportements de ce groupe. L'excessive marginalisation des Amhara entraîna des phénomènes de résistance, notamment au sein de l'administration, et mit en difficulté plusieurs fois une politique dont la réalisation nécessitait une mobilisation importante des capacités intellectuelles et techniques de l'appareil d'Etat. Les cadres du Parti des travailleurs éthiopiens demeurent le plus souvent l'objet d'une grande méfiance durant cette phase intérimaire alors que sociologiquement ils représentent une strate sociale absolument indispensable pour la mobilisation des forces vives du pays ${ }^{19}$.

Une dernière raison était liée à l'obligation internationale pour le FDRPE de coopter le plus rapidement des organisations ayant une autre expression politique au niveau de l'Ethiopie afin de donner au moins l'apparence de la démocratie. Ceci fut fait une première fois d'une main de maître lors de la conférence nationale en juillet 1991. Le FDRPE intégra dans le jeu politique national des organisations qui prétendaient représenter un groupe ethnique sans s'interroger sur leur légitimité pour peu qu'elles acceptent des compromis sur leurs revendications et ne remettent pas en cause la position absolument dominante (qu'on pense à la composition actuelle des forces armées) du FPLT. Le but visé était donc d'obtenir d'une part une reconnaissance politique de la part de groupes ethniques ou régionaux qui n'avaient pas été réellement défaits dans la guerre et, d'autre part, une déconnexion entre des revendications "admissibles" puisque pouvant trouver une réponse à un niveau régional et d'autres qui affecteraient d'emblée l'économie du pouvoir au plan national et donc l'hégémonie du FDRPE. C'est cette stratégie qui a été mise en application avec un peu trop d'enthousiasme par les cadres du FDRPE et de quelques partis épigones lors des élections régionales de juin 1992; malheureusement pour eux, les observateurs internationaux semblaient avoir une conception différente des élections pluralistes et le firent savoir aux Etats-Unis qui étaient disposés dans un premier temps à passer l'éponge sur des pratiques plus que contestables. Une question irrésolue jusqu'à aujourd'hui concerne le rôle qu'entend jouer dans le futur sur la scène politique le Parti démocratique révolutionnaire du peuple éthiopien (PDRPE), fondé lors du congrès du FDRPE en janvier 1991, qui s'efforce de recruter à l'heure actuelle de façon discrète, mais relativement systématique, dans toutes les organisations nationalitaires. Certes le FDRPE a réaffirmé plusieurs fois sa conversion au multipartisme et à la démocratie, 
mais cette démarche est parallèle à la stratégie antérieure du FPLT construite autour d'un "front uni large et démocratique" (qui pourrait être aujourd'hui formé des organisations présentes à la Conférence nationale) et d'un "front uni stratégique révolutionnaire" (hier les ligues marxistes-léninistes, aujourd'hui le PDRPE).

Le cas des Oromo est dans un tel dispositif tout à fait paradigmatique ${ }^{20}$. Ils constituent plus de $40 \%$ de la population du pays (certains affirment qu'ils seraient même majoritaires) et sont musulmans à $60 \%$, orthodoxes à $30 \%$, le reste se partage entre des églises missionnaires comme Mekane Yesus et l'adhésion à la religion "traditionnelle" des Oromo. Ils peuvent revendiquer donc le territoire le plus important de l'Ethiopie ainsi que les deux premières villes du pays, Addis-Abéba (Finfine en oromo) et DireDawa. Certes, les organisations oromo ont fait de notables compromis en octobre 1991 au moment de la discussion des nouvelles entités régionales qui devaient servir de cadre aux élections au début $1992^{21}$; ces dernières furent d'ailleurs reportées plusieurs fois pour se tenir finalement en juin dans des conditions telles qu'elles furent boycottées par plusieurs organisations oromo, dont le FLO. Evidemment, de telles aspirations territoriales étaient contestées par d'autres groupes quelquefois violemment comme ce fut le cas plusieurs fois durant l'années 1991 et 1992 pour DireDawa, puisque, s'il est souvent délicat de définir une frontière d'un territoire ethnique, l'identité d'une ville selon un tel critère demeure encore bien plus problématique: affrontements en juilllet, août, septembre, novembre, puis pratiquement durant tout l'hiver $91-92^{22}$ et qui fut apparemment négocié sous pression. Cependant quatre organisations oromo ont obtenu une représentation au Parlement (ou Conseil des représentants) et deux au gouvernement; trois de ces organisations méritent d'être mentionnées ici $^{23}$. La plus importante des quatre, celle qui a conçu le corpus idéologique et politique le plus sophistiqué est sans conteste le FLO. Créé en 1974, actif après 1978 au Soudan dans l'organisation des réfugiés qui avaient fui pour beaucoup la conscription forcée, il débuta ses actions militaires dans le Wollega en 1982-1983; le nombre de ses combattants est passé de quelques centaines à cette époque à plus de 10.000 en 1990-91. Cependant, au moment de la préparation des élections régionales de juin 1992, il ne fut pas capable malgré une campagne de recrutement forcé sur plusieurs semaines d'atteindre le chiffre de 40.000 combattants comme il l'avait annoncé lors des négociations sur le cantonnement de ses troupes ${ }^{24}$. Le FLO disposait de quatre ministères au gouvernement (Information, Agriculture, Education nationale, Commerce) qu'il abandonna le 23 juin 1992 pour protester contre les conditions dans lesquelles s'étaient déroulées les élections. Depuis cette date, le FDRPE a emprisonné dans des camps près de 17.000 membres (civils et combattants) du FLO ${ }^{25}$, compte tenu du climat d'hostilité et de l'impasse des négociations entre les deux organisations, malgré une habile médiation érythréenne. Le Front islamique de libération de Oromiyya (pays oromo), -FILO- a été créé en 1986 à partir d'une scission du FLO datant de 1978 et a alors adopté ce nom essentiellement pour bénéficier les faveurs de l'internationalisme islamique, de là à en conclure qu'il s'agit d'une organisation islamiste il y a un pas qu'il ne faut pas franchir ${ }^{26}$

29 . L'organisation démocratique du peuple oromo (ODPO) est un mouvement affilié au FDRPE, largement créé au début 1990 à partir de prisonniers de guerre du FPLT retournés. Profitant largement de l'appui du FDRPE et du sentiment légitimiste des cadres ruraux, cette organisation a pu prendre pied dans le Wollega, l'Ilubabor et les bourgs du pays oromo. Partageant pour l'essentiel la perception du FDRPE sur la question oromo, elle a tenté avec plus ou moins de succès de contrecarrer la 
progression du FLO dans les campagnes, provoquant d'ailleurs des affrontements. Pourtant, il semble que dans les derniers mois, désireuse de conforter certains succès remportés en juin ou de ne pas s'isoler davantage par son alliance avec un pouvoir très contesté par les Oromos, elle ait pris des positions moins suivistes et se soit rapprochée du FLO, provoquant d'ailleurs des réactions d'hostilité du FDRPE. L'ODPO n'en conserve pas moins ses deux ministères (Intérieur et Affaires sociales) et son groupe parlementaire au Conseil des représentants.

30 La divergence fondamentale entre FLO et FDRPE peut s'énoncer très simplement: la question oromo relève-t-elle d'une interprétation en terme de féodalisme (et dans ce cas la conservation de la structure étatique ne fait plus problème) ou de colonialisme (dans ce cas l'éclatement est synonyme de libération à mois que...). Plutôt que d'essayer de trancher un débat aussi byzantin, quand bien même on peut s'accorder sur son importance, il vaut peut-être mieux comprendre l'organisation de la conception du nationalisme telle qu'elle est développée par le FLO. Il faudrait certes analyser les raisons qui ont permis à une partie non négligeable de la population oromo de s'identifier aux thèses nationalistes, au-delà de soulèvements locaux ponctuels au milieu des années 1970: maintien d'une politique de bas prix aux producteurs pour maintenir un approvisionnement des villes à bon marché malgré une réforme agraire dont les premiers mois avaient été perçus avec beaucoup de sympathie, réinstallation autoritaire de populations sur des territoires oromos sans consultation des autorités locales, villagisation, conscription forcée, répression politique, effets différés de la guerre de l'Ogaden en 1977-78.

31 La pierre angulaire de la construction de l'identité nationale est depuis dix ans - faut-il s'en étonner ?- le discours sur la culture oromo avec notamment un usage de la langue écrite en caractères latins et l'idée d'une contre-société projetée dans un passé idéalisé où tout était pour le mieux dans le meilleur des mondes jusqu'à l'arrivée de Menelik à la fin du siècle dernier. On oublie le rôle des Oromos du Shoa qui constituèrent les forces supplétives de la "colonisation" amhara, la cooptation d'élites oromo locales, le système d'inégalités qu'engendre le système gadaa de classes d'âge. Dans le même temps, le discours nationaliste s'est efforcé de définir des rapports très théoriques entre la nation oromo et les autres groupes présents sur le territoire qui serait le sien ; il y a d'abord les nations voisines qu'on distingue parce qu'elles possèdent une langue, une culture, une histoire, un territoire propre ; ensuite les nationalités (i.e. les petites nations) qui vivent sur des territoires revendiqués comme oromos et possèdent une langue, une culture et une histoire (comme les Adare de la ville d'Harrar); enfin les étrangers qui se subdivisent en deux : les colons (naftanyoota, qui devraient retourner chez eux), les migrants comme les Somali ou les Gurage, les Grecs ou les Arméniens qui peuvent rester à condition d'accepter la constitution mais qui ne sont pas réellement reconnus comme communautés.

32 La dynamique conflictuelle actuelle s'inscrit cependant à plusieurs niveaux qui ne sont pas réductibles les uns aux autres. D'abord, il y a bien sûr une compétition politique entre les nationalistes oromo et le pouvoir central qui est ancienne mais qui a aujourd'hui des enjeux qui ne sont pas purement idéologiques. La composition des forces armées, la nomination des administrateurs locaux, le contrôle des mécanismes de décision au niveau gouvernemental constituent à ce niveau des points de clivage essentiels. Cependant, il faut également mentionner la très importante composante sociale dans les troubles actuels. Ceux-ci ont très schématiquement une double origine. 
D'abord, le gouvernement éthiopien a entrepris une démobilisation aussi radicale que rapide de l'ancienne armée. Près de 350.000 hommes, peut-être plus, se sont retrouvés pratiquement du jour au lendemain sans ressource et certains se sont reconvertis dans le banditisme rural dans la grande tradition des shifta du XIXème siècle. Mais l'autre aspect est sans doute essentiel. A la faveur de la propagande nationaliste, la paysannerie oromo a relancé les mouvements de contestation qui s'étaient déjà exprimés une première fois entre 1974 et 1976 puis avaient été gelés par la répression du pouvoir central. Derrière les massacres d'Amhara, il y a souvent plus qu'une question ethnique ou religieuse mais également des luttes foncières dont les enjeux sont accrus par la nouvelle politique de libéralisation de l'agriculture. Enfin, il faut mentionner, comme dans toute construction d'une idéologie nationaliste, derrière l'unanimisme de certaines références (comme l'officialisation de l'usage de la langue oromo ou son écriture), la compétition entre organisations où se manifestent le poids des influences extérieures et la fragilité de la construction d'une identité nationale. Ainsi les affrontements entre FLO et ODPO renvoient moins pour l'instant à des différences régionales (dans les deux cas la direction politique des organisations est originaire du Wollega et de confession chrétienne) qu'au mode d'accès à l'Etat et aux alliances à conclure avec les notables ruraux. La différence à l'intérieur des Oromos entre chrétiens et musulmans n'a pas encore provoqué de réelle confrontation, mais il est probable que l'interventionnisme de certains Etats voisins aura des conséquences néfastes dans les mois qui viennent, surtout si les tentatives de médiation tournaient court et que la confrontation avec le pouvoir central reprenait une tournure violente.

La question oromo autant que la trajectoire de l'APLS reposent sous une forme très complexe une série de questions dont les réponses affecteront l'ensemble des trajectoires politiques des Etats de la région. Le Soudan n'accepterait pas sans broncher une nouvelle politique éthiopienne vis-à-vis de l'APLS et jouerait alors de son influence chez certains partis oromo mais aussi chez d'autres organisations nationalitaires en Ethiopie. De la même manière, une confrontation trop violente entre Oromo et Somali, après toute une période d'alliance des deux nationalismes ${ }^{27}$, risquerait également de provoquer de nouvelles déflagrations dans le sud de l'Ethiopie, d'autant que les armes ne manquent guère depuis l'intervention américaines en Somalie. Mais la question la plus importante est celle liée à l'articulation entre conflits sociaux, aspirations et statuts territoriaux: cette dimension qui met l'accent sur les acteurs locaux et déconstruit les rationalités globales qui servaient de cadre au moins apparent aux conflits dans la période antérieure possède une dynamique propre dont les effets politiques risquent d'être plus délicats encore à gérer. Le cas de Djibouti est à ce niveau aussi exemplaire que dramatique : la polysémie de la territorialité permet de justifier l'action d'un Etat autoritaire autant que les prétentions quelquefois excessives des Afar, dont certains aspirent à une représentation qui diluerait un peu vite l'importance de la démographie dans celle de la superficie des territoires revendiqués. Si l'on voulait être plus optimiste, on pourrait affirmer que la Corne sera le lieux des scénarios croisés du nouvel ordre mondial en Afrique. Mais jusqu'où cet ordre impliquera-t-il la fin des désordres armés et la recomposition des sociétés civiles à l'opposé de la militarisation des champs politiques des dernières années? 


\section{NOTES}

1. Pour en rester à une vision purement statistique dans le cas éthiopien : selon le ministre de la Défense du gouvernement provisoire éthiopien, Siye Abraha, il ressort de documents officiels des gouvernements éthiopiens depuis 1974 que l'armée éthiopienne qui comptait 38.815 militaires en 1974 a recruté 1,1 million de personnels durant ces 17 années. L'ancien régime a acquis 370 avions (dont 224 avions de guerre et hélicoptères de combat), plus de 1700 tanks, 1600 autres engins blindés et 4000 pièces d'artillerie. Il a reconnu officiellement 300.000 pertes humaines avant l'offensive finale du FDRPE. Mais ce dernier estime les pertes à plus de 500.000 morts pour la seule armée régulière. Le ministre de la Défense a estimé que les pertes du côté des insurgés avaient été cinq fois moins importantes. Les pertes civiles sont estimées à un million de mort. En 1991, le budget militaire de l'ancien régime (plus de 2,2 milliards de birr) était 20 fois supérieur à celui de 1974. En 17 ans, les dépenses militaires ont représenté 17 milliards de birr et les achats d'armement environ 18 milliards de birr au total 35 milliards de birr soit environ 17 milliards de dollars, Lettre de l'Océan Indien, 16/11/91

2. Roland Marchal " Conflits et recomposition d'un ordre régional dans la Corne de l'Afrique " Etudes internationales, vol. XXII, n², juin 1991, pp. 307-321.

3. Bien que cette information ait été démentie plusieurs fois par les principaux intéressés, de nombreux observateurs s'accordent à penser que des troupes d'élite érythréennes ont joué un rôle clef dans la prise de la capitale éthiopienne par le FDRPE. De plus, l'aide logistique et les instructeurs du FPLE furent également indispensables à l'autre composante de l'opposition éthiopienne active jusqu'en juin 1991 essentiellement dans le Wollega, le Front de libération Oromo (FLO).

4. Voir le dossier publié par Politica Internazionale, $n^{\circ} 4$, juillet-août 1992 , avec notamment mon article "Il caso eritreo", pp. 67-79.

5. Voir le dossier publié par Politica Internazionale, $n^{\circ} 4$, juillet-août 1992 , avec notamment mon article "Il caso eritreo", pp. 67-79.

6. Pour une présentation du conflit sud-soudanais, on pourra se reporter notamment à : Abel Alier, Southern Sudan : too many agreements dishonoured, Exeter, Ithaca Press, 1990 et Roland Marchal, "D'une guerre à l'autre : le conflit au Soudan hier et aujourd'hui", Afrique contemporaine, n 153 , 1er trimestre 1990, pp. 27-42.

7. Cette remarque vaut pour toutes les organisations armées de la région. Le FPLE et le FLO notamment ont su acquérir un soutien et une audience au-delà de leurs cercles habituels grâce à ce média dont l'utilisation par les mouvements armés devraient susciter une analyse plus circonstanciée.

8. Voir Roland Marchal, "Le Soudan entre islamisme et dictature militaire", MaghrebMachrek, n¹37, juillet-septembre 1992, pp...56-80.

9. Entretiens avec les représentants de ces mouvements à Londres et à Khartoum (octobre-novembre 1990).

10. Thomas Zitelmann, "Politische Grenze und strategisches Hinterland - Überlegungen zur grenzüberschreitenden Mobilität zwischen dem Sudan und Äthiopien " in Bernhard Streck (ed), Tradition, Migration, Notstand - Themen heutiger Sudanethnologie, Göttingen, Ed. Re, 1990, pp. 97-109.

11. Cette intervention des troupes éthiopiennes dans le conflit sud-soudanais n'a sans doute guère cessé depuis. De nombreuses rumeurs font état d'une implication 
éthiopienne (oromo ?) et même érythréenne dans les combats sur la zone frontalière, par exemple pour la prise de Pochalla en mars 1992, pour le plus grand avantage de Khartoum qui obtient ainsi les dividendes de son aide massive aux nouveaux dirigeants de l'Ethiopie et de l'Erythrée. La Lettre de l'Océan Indien, 02/05/92 \& Africa Analysis, $30 / 10 / 92$.

12. Voir la nouvelle édition qui comporte en outre des documents plus récents particulièrement intéressants sur la crise du mouvement : Mansour Khalid (ed..), The Call for democracy in Sudan by John Garang, Londres, Kegan Paul International, 1992. 13. Lam Akol, Shilluk, enseignant à l'Université de Khartoum et docteur ingénieur, avait été élu député aux élections d'avril 1986. Surtout, il fut à la même époque l'un des négociateurs de l'accord mort-né de Kokadam entre l'APLS et une majorité de partis soudanais dont le parti Umma de Sadeq el-Mahdi. Il passa à la guérilla en mai 1986 lorsque ce dernier, devenu premier ministre, refusa d'appliquer l'accord et revint sur des promesses faites durant la campagne électorale dont la réalisation aurait considérablement détendu le climat entre les insurgés et Khartoum. Gordon Koang Chol, quant à lui, est un Nuer et a fait ses premières armes dans la guérilla des années 1960 mais ne put intégrer l'armée soudanaise en 1972 pour des raisons de santé et devint un petit employé. En 1982, il gagna l'Ethiopie où résidait une partie de sa famille et s'engagea alors dans Anya-nya II, dont il prit la direction quelques années plus tard. C'est lui qui négocia l'accord de 1988 avec l'APLS, qui mit pratiquement fin pour trois ans à une guerre entre sud-Soudanais dont les victimes se comptent par milliers. Il représente la véritable force militaire de la dissidence.

14. Voir Africa Analysis, 21/08/92.

15. Voir Sudan Democratic Gazette, décembre 1992

16. Voir Amnesty International, Soudan : morts et incarcérations : la destruction de Juba., 23 septembre 1992. C'est l'exécution de deux employés l'un travaillant pour USAID et l'autre pour la CEE qui a déclenché une campagne internationale nourrie depuis par les multiples exactions commises contre la populations par les deux protagonistes armés. Voir Amnesty International, " Sudan : both sides committing grave abuses in the South says Amnesty International", Weekly Update News, 04/11/92. 17. Albert Rimbaud in Hérodote 18. Voir Randi Ronning Balsvik, Haile Sellassie's students : the intellectual and social background to revolution, 1952-1977, East Lansing, African Studies Center Michigan State University, monograph $n^{\circ} 16,1985$.

19. Voir l'analyse qu'en propose Christopher Clapham dans son ouvrage, Transformation and continuity in revolutionary Ethiopia, Londres, Cambridge University Press, 1988.

20. Voir John Markakis, National and Class Conflict in the Horn of Africa, Cambridge, Cambridge University Press, 1987 et Gadaa Melbaa, Oromia, Khartoum, 1988 (pas de maison d'édition) pour une vision plus militante.

21. Africa Confidential, vol. $32, n^{\circ} 22$,

22. Pour une chronique plus ou moins exhaustive et exacte, se reporter à la Lettre de l'Océan Indien durant cette période.].

Cependant l'importance que revêt la question oromo aujourd'hui n'est pas simplement liée à la superficie du territoire de cette nationalité, pour reprendre cette catégorie du discours politique éthiopien, mais également à l'affirmation d'un droit à la sécession qui mettrait en cause radicalement l'existence de l'Etat éthiopien et la pérennité de l'Ethiopie. Sans doute, les mouvements politiques oromo ont-ils des opinions plus ou 
moins nuancées sur cette question, diversité qui peut s'expliquer autant par leur compétition que leur recrutement, mais, par certains aspects, la dynamique du débat depuis un an poussent à une homogénéisation des points de vue, voire sur des questions très précises à une radicalisation. Même si certaines positions sont susceptibles d'évoluer, des désaccord fondamentaux n'en continuent pas moins d'exister avec le nouveau pouvoir en place, liés fondamentalement à la quasimonopolisation de la gestion du pays par les Tigréens, qui sont comme les Amhara perçus par les Oromo comme les descendants des colonisateurs abyssins.

Il est également intéressant de noter qu'au moment de la Conférence nationale d'AddisAbeba en juillet 1991, les cinq organisations oromo présentes furent au préalable réunies par le gouvernement de Khartoum, qui réussit à les convaincre de signer un accord qui n'eut pas de suite[[Voir la Lettre de l'Océan Indien 01/06/91 et Africa Confidential, vol32. $\mathrm{n}^{\circ} 4,12 / 07 / 91$.

23. Les deux autres sont les suivantes. la Direction unie du peuple oromo (United Oromo people's Leadership) a été fondée deux jours avant la conférence nationale d'Addis-Abéba et est composée pour l'essentiel d'anciens membres du Front de libération somali-abo. Son leader est une figure historique du mouvement de rebellion dans le Balé au milieu des années 60 : Waaqo Guutu Usu (voir outre l'ouvrage déjà cité de John Markakis, l'étude magistrale de Gebru Tereke, Ethiopia : power and protest, Cambridge, Cambridge University Press, 1991). Le second mouvement est le Front de libération oromo-abo, issu de la même organisation que le précédent. Son dirigeant est Siraaj Haaji Isaaq, qui fut le leader d'un mouvement rural autonomiste "Bilisa Baaftu Oromoo" en 1975-76 dans le Balé.

24. Information recueillie lors de la mission d'observation des élections dans la région de Dire-Dawa en juin 1992

25. Ces camps sont visités régulièrement par le CICR ; pour l'heure, il n'y aurait aucun manquement au respect du droit international concernant la détention de ces personnes, si l'on excepte évidemment l'absence de procédures judiciaires...

26. Son dirigeant est Sheck Jaara, de son vrai nom Abdulkarim Ibrahim Hamid, qui fut un cadre du Western Somali Libération Front entre 1960 et 1969 avant de passer quelques années dans les geôles de Siyaad Barre. De façon assez significative, la division de 1978 fut entre la "branche de Khartoum" et la "branche de l'Est" du FLO : elle rendait compte de la diversité sociologique impressionnante des Oromo autant que de facteurs politiques plus traditionnels comme l'influence des Etats les plus proches ou les luttes pour le pouvoir.

27. Il ne s'agit pas d'ignorer l'instrumentalisation des Oromos par l'Etat somalien indépendant mais cette politique pratiquement constante depuis l'indépendance n'épuise pas l'analyse de cette question.

\section{RÉSUMÉS}

La fin de la bipolarité, à savoir l'effondrement du système soviétique n'a pas joué dans la corne de l'Afrique comme un facteur d'atténuation voire même de disparition de la conflictualité. Les 
situations régionales, et plus particulièrement celles de Ethiopie, du Soudan seul véritable bénéficiaire au niveau local de la chute du régime de Mengistu, de la Somalie, et même de Djibouti, doivent selon Roland Marchal s'analyser plus comme les conséquences d'un phénomène de résonances entre des logiques internes et internationales que comme le résultat d'une induction par l'extérieur, quel que soit le rôle joué par la faiblesse ou la modération soviétique.

The collapse of the Soviet system and the end of bipolarity have not contributed to peace in the Horn of Africa. Regional developments - whether in Ethiopia, Sudan (the only country in the region that really benefited from the fall of Mengistu), Somalia or even Djibouti - continue being influenced, not so much by external factors and the role played by the Soviets, than by a phenomenon of internal and international resonances.

\section{INDEX}

Mots-clés : conflits, sortie de conflits

Index géographique : Corne de l'Afrique 